\title{
PRINCIPALES CONCLUSIONES ACORDADAS DURANTE LA CUMBRE SOBRE DISCAPACIDAD INTELECTUAL Y DEMENCIA
}

\section{Main conclusions agreed during the Summit on Intellectual Disability and Dementia}

Matthew P. JANICKI

University of Illinois at Chicago, USA

Karen WATCHMAN

University of Stirling, Scotland, UK

Juan ForTEA ORMAEChEA

Centro Médico de Down de la Fundació Catalana de Síndrome de Down (FCSD) y Servicio de Neurología del Hospital de la Santa Creu i Sant Pau

jfortea@santpau.cat

Y miembros del International Summit on Intellectual Disability and Dementia

$\mathrm{E}$

STE BREVE INFORME resume las recomendaciones de la Cumbre Internacional sobre Discapacidad Intelectual y Demencia, celebrada en Glasgow, Escocia, del 13 al 14 de octubre de 2016, organizada por la Universidad de Stirling y la Universidad del Oeste de Escocia, financiada por el RS MacDonald Trust, el Gobierno escocés y Alzheimer Scotland. Los patrocinadores colaboradores incluyeron el Grupo de Trabajo Nacional sobre Discapacidades Intelectuales y Prácticas de Demencia (NTG) en los Estados Unidos y la Universidad de Illinois en Chicago. La cumbre fue copresidida por Karen Watchman, Ph.D. y Matthew P. Janicki, Ph.D., y tuvo representantes multidisciplinares de distintas organizaciones nacionales e internacionales con intereses en cuestiones relacionadas con los adultos con discapacidad intelectual afectados por demencia. El contenido de esta declaración fue parcialmente desarrollado gracias a una subvención del Departamento de Salud y Servicios Humanos de los Estados Unidos, Administración para la Vida Comunitaria (ACL), Beca 
del Instituto Nacional de Discapacidad, Vida Independiente e Investigación en Rehabilitación (NIDILRR) \# 90RT5020-03-00. Sin embargo, esos contenidos no representan necesariamente la política del Departamento de Salud y Servicios Humanos de EE. UU., ni el respaldo del Gobierno Federal de EE. UU. Las opiniones expresadas representan las de los participantes de la Cumbre y del NTG. 2 Miembros de la Cumbre Internacional: Cathy Asante (Escocia), Anna Beránková (República Checa), Kathleen Bishop (EE. UU.), Nicole Cadovius (EE. UU.), Sally-Ann Cooper (Escocia), Tonni Coppus (Países Bajos), Jim Crowe (Gales ), Karen Dodd (Reino Unido), Juan Fortea (España), Claudia Gaertner (Alemania), Kiran Haksar (Escocia), Flavia Heloisa Santos (Brasil), Mary Hogan (EE. UU.), Matthew P. Janicki (EE. UU.), Nancy Jokinen (Canadá), Seth Keller (EE. UU.), Frode Larsen (Noruega), Ronald Lucchino (EE. UU.), Philip McCallion (EE. UU.), Mary McCarron (Irlanda), Peter Mittler (RU), Jim Pearson (RU), Sam Quinn (Escocia), Anne-Sophie Rebillat (Francia), Evelyn Reilly (Irlanda), Kathy Service (EE. UU.), Michael Splaine (EE. UU.), Sandy Stemp (Canadá), Andre Strydom (Reino Unido), Gomiero Tiziano (Italia), Leslie Udell (Canadá), Karen Watchman (Escocia).

Durante la reunión internacional celebrada en Glasgow, Escocia, en 2016, se examinó la problemática asociada a la demencia en los adultos con discapacidad intelectual (DI). La temática abordada y los problemas estudiados incluyeron: (1) los derechos bumanos y recursos fundamentales de las personas (aplicaciones de la convención de los derechos de las personas con discapacidad y del derecho a la inclusión social y perspectivas de las personas con DI); (2) servicios individualizados y apoyo clínico (a la progresión de la demencia y demencia avanzada, soporte humano tras el diagnóstico, ayudas sociales en el contexto comunitario y servicios, experiencia, capacidad y conocimiento acerca de personas que padecen de demencia, de los cuidados específicos que requieren, de la metodología de dichos cuidados y del acompañamiento al final de la vida), y (3) amparo y defensa de las conclusiones consensuadas, impacto público, soporte al cuidador/familiar (nomenclatura/terminología, inclusión de la problemática de las personas con DI en los planes nacionales y cooperación con el cuidador/familiar). Los resultados incluyeron una serie de artículos con información y recomendaciones sobre las temáticas mencionadas. Estos artículos tienen como objetivo asistir a las diferentes organizaciones y agentes cuyo proyecto ofrece amparo y cuidados destinados a este grupo poblacional, como también a las administraciones gubernamentales en la planificación de estos cuidados específicos dirigidos a personas con DI, así como la protección, sostenimiento y difusión en favor, y en nombre, de las personas con DI afectadas por demencia y de sus familias.

\section{Introducción}

El interés actual sobre la problemática asociada a la demencia entre los adultos con discapacidad intelectual (DI) se deriva de estudios de investigación que muestran un riesgo más elevado y un inicio más temprano de demencia entre algunos adultos con DI, particularmente aquellos con síndrome de Down; el envejecimiento de la 
población con DI, y la manifiesta falta de preparación por parte de administraciones gubernamentales y organizaciones que proveen servicios especializados relacionados con la demencia en adultos con DI. Dado el aumento del número de adultos ancianos con DI, debido tanto al aumento de la esperanza de vida de esta población como por los efectos del "baby boom" (la cohorte de sujetos nacidos entre mediados de 1940 hasta mediados de 1960, época en que se produjo una alta tasa de natalidad), como la inclusión de la DI en el informe de la Organización Mundial de la Salud sobre la demencia (OMS, 2012, 2017), se estimó oportuno realizar una revisión de los problemas emergentes en el ámbito de la asistencia social en este grupo de personas. Con esta finalidad, se convocó una cumbre internacional sobre discapacidad intelectual y demencia en Glasgow, Escocia, del 13 al 14 de octubre de 2016. El objetivo de la Cumbre fue examinar e informar sobre el estado del conocimiento en varios temas poco investigados en relación a la demencia y la DI, incluyendo la definición de demencia avanzada y el final de la vida, las estructuras de apoyo tras el diagnóstico, las necesidades y soporte al familiar o cuidador, una planificación proactiva y las perspectivas subjetivas sobre la atención y el impacto de la demencia (Vigilante et al., 2017b). Los informes de la Cumbre fueron diseñados con el objetivo de ser útiles para los profesionales, coordinadores de acciones y recursos, equipos de apoyo y defensa, así como organizaciones gubernamentales, no gubernamentales o del tercer sector, con la idea de proponer y promover un consenso en los objetivos y metodología de cada uno de los proyectos multidisciplinares alrededor del cuidado transversal de las personas con DI en un futuro. Se examinaron tres áreas temáticas principales, que incluyen (1) Derechos humanos y recursos personales (aplicaciones de la Convención para los Derechos de las Personas con Discapacidad y derechos humanos a la inclusión social y perspectivas de las personas con DI); (2) Servicios individualizados y clínicos de apoyo (progresión de la demencia y demencia avanzada, apoyo tras el diagnóstico, soporte y recursos sociosanitarios en la comunidad, la capacitación alrededor de la atención a personas con DI en su día a día , así como metodología común en los cuidados y acompañamiento a estas personas durante el final de su vida), y (3) Promoción, impacto público y temas en torno al cuidador familiar (terminología y uso del lenguaje, inclusión de personas con identificación en los planes nacionales y el amparo a los cuidadores familiares).

A continuación se resumen estos contenidos y se enuncian las principales recomendaciones.

\section{Derechos humanos y recursos personales}

Derechos Humanos y la Convención para los Derechos de las Personas con Discapacidad. Dentro del campo de la DI, hay una convocatoria para fomentar la consideración de la demencia como un área de servicios especializados, para que las personas con demencia reciban una adecuada consideración bajo la Convención de las $\mathrm{Na}$ ciones Unidas para los Derechos de las Personas con Discapacidad (CDPD, y para una mayor interacción y aprendizaje compartido entre los servicios de atención a la 
demencia y a la discapacidad, puesto que un enfoque unificado tiene el potencial de ofrecer más opciones para tratar los problemas relacionados con la demencia a los que se enfrentan las personas con DI. Se hicieron tres recomendaciones: (a) reconocer la demencia como una limitación social bajo la CDPD, (b) apoyar un enfoque de derechos humanos para la autodeterminación cuando una persona con DI se ve afectada por una demencia y (c) apoyar un mayor diálogo y cooperación entre los servicios de apoyo y atención a la DI y la demencia.

Perspectivas de las personas con discapacidad intelectual. La perspectiva de las personas con demencia en la población general se conocen cada vez mejor por el impulso en la participación e implicación de estas en los procesos; hay muy poco conocimiento sobre estas perspectivas en las personas con DI, lo que limita la comprensión de su experiencia de la demencia y el diseño de los servicios de apoyo necesarios (Watchman et al., 2017a). Se hicieron tres recomendaciones: (a) promover la investigación sobre situaciones que requieren estándares de cuidado; (b) permitir una mayor participación de los propios afectados en el diálogo con los proveedores, y (c) aumentar los esfuerzos para eliminar los prejuicios que persistan en los comités éticos y de investigación sobre la capacidad de las personas con DI de ser informadores en los estudios.

\section{Servicios individualizados y apoyos clínicos}

Demencia avanzada. Diagnosticar la demencia avanzada en personas con DI es más complicado que en la población general debido al variable nivel de DI preexistente y a una mayor presencia de comorbilidades o comportamientos que a menudo imitan los síntomas de la demencia avanzada (McCallion et al., 2017). Es necesario saber cuándo está presente la demencia avanzada, ya que las necesidades y respuestas de atención diferirán de las presentes en la etapa temprana de asistencia, entre las que se cuentan los cuidados y acompañamiento al final de la vida. Se hicieron tres recomendaciones: (a) apoyar la evaluación continua de los cambios en la progresión de la enfermedad; (b) alentar la investigación dirigida a identificar herramientas clínicas más sensibles para reconocer la progresión en los estadios más avanzados o la demencia avanzada, y (c) desarrollar una mejor capacitación en el cuidado de la demencia avanzada, así como guías de práctica clínica.

Amparo tras el Diagnóstico. Este apoyo comienza tras el diagnóstico inicial de demencia, e implica explicar el diagnóstico y hablar sobre los cambios de salud (si se estima más apropiado), así como promover la toma de decisiones por parte del paciente sobre la asistencia y apoyo futuros, y generalmente adaptar los apoyos a la situación de cada individuo y a la naturaleza de la DI, así como con las intervenciones de apoyo tras el diagnóstico (ATD) para los síntomas idiosincrásicos conductuales y psicológicos de la demencia (SCPD) (Dodd et al., 2017). La planificación del ATD comienza en el momento en el que se confirma el diagnóstico y continúa hasta el final de la vida de la persona. Se hicieron tres recomendaciones: (a) estudiar la efectividad de las diferentes intervenciones no farmacológicas; (b) desarrollar guías de práctica 
clínica para el ATD para los cuidadores y personal de apoyo; (c) investigar la prevalencia y naturaleza de los SCPD en los adultos con DI que desarrollan demencia.

Amparo a la Demencia en el ámbito comunitario y social. Cuando progresa la demencia, es preferible promover la inclusión social del alojamiento específico, junto a la necesidad personal competente con una capacitación continua en el cuidado de la demencia. Este tipo de vivienda especializada para personas con la demencia debería incluir sostenibilidad, adaptación ambiental, respuesta a las necesidades individualizadas y un enfoque dirigido a cuidados de calidad, enfoques centrados en la persona e integración en la sociedad. Las opciones menos favorables (como centros sociosanitarios) rara vez se justifican, ya que pueden alterar las rutinas establecidas y las relaciones sociales, propiciar la pérdida de estructura familiar y comprometer las relaciones personales con familia y amigos. Se hicieron tres recomendaciones: (a) desarrollar estándares de cuidado para servicios basados en la comunidad que proporcionan vivienda y otros apoyos para personas con DI y demencia; (b) promover entornos de vida con demencia en todos los lugares llamados 'hogar', y (c) prevenir cambios arbitrarios en la residencia por mandato de las autoridades gubernamentales.

Cuidado al final de la vida. Llegado el momento en que la demencia está avanzada, la atención física debe estandarizarse con un enfoque en el manejo del dolor, la comodidad y el alivio de las condiciones coincidentes (McCarron et al., 2017a, b; Service et al., 2017). Se deben ofrecer servicios de cuidados paliativos y fomentar acuerdos de cooperación entre los susodichos proveedores de servicios paliativos y los servicios de apoyo a las personas con DI y sus familias con el fin de aumentar su utilización. Se debe aclarar el concepto de "apoyo activo" al final de la vida y se deben desarrollar pautas avanzadas de planificación de la atención que reflejen las distintas visiones ideológicas y socioculturales en torno al final de la vida, así como el consentimiento y el estatuto legal que permita la planificación. Se hicieron tres recomendaciones: (a) crear una guía de práctica universal sobre los apoyos al final de la vida; (b) alentar el uso de tales apoyos para la atención al final de la vida en el hogar, y (c) reconocer variaciones en lo que "hogar" puede ser en relación a la atención al final de la vida.

\section{Defensa, impacto público y problemas del cuidador familiar}

Terminología y uso del lenguaje. El uso del lenguaje relacionado con la DI y la demencia carece a menudo de precisión (Janicki et al., 2017). La investigación clínica o médica relacionada con la DI y la demencia generalmente contiene definiciones estructuradas de demencia o términos relacionados, pero el lenguaje en los artículos de atención social tiende a una menor precisión. Esto se debe a una falta de comprensión de la distinción entre los diferentes tipos de demencia, el uso inconsistente del lenguaje y/o el resultado de la ausencia de métodos y criterios de consenso para el diagnóstico. La consistencia en la terminología puede ayudar a armonizar los protocolos y a estudiar las comunicaciones de procedimientos y resultados. Se hicieron tres recomendaciones: (a) adoptar una lista estandarizada de términos para uso general por parte de agentes e investigadores; (b) estandarizar los informes para incluir los 
demográficos y otra información clave de los sujetos, y (c) promover imágenes positivas a través de un lenguaje no estigmatizador.

Inclusión en planes y estrategias nacionales de demencia. La OMS pidió el desarrollo y la adopción de planes o estrategias nacionales para orientar las políticas públicas y establecer objetivos para los servicios, los apoyos y la investigación relacionados con la demencia; dichos planes o estrategias deben incluir aspectos concretos en relación a los problemas, las necesidades y las respuestas para apoyar a los adultos con DI, y dicha inclusión debe ir más allá de la mera descripción y reconocimiento de la relevancia del problema (Watchman et al., 2017c). Los adultos con DI o sus gestores deben participar en cualquier proceso que produzca dichos documentos. Se hicieron tres recomendaciones: (a) incluir adultos con DI en los procesos de redacción de los planes nacionales; (b) abogar por que los gobiernos proporcionen información en relación a la DI que apoye el desarrollo de dichos planes, y (c) involucrar a los gestores en el desarrollo o revisión de las políticas y de los planes.

Cuidadores familiares. Las familias son la principal fuente de alojamiento y apoyo para los adultos con DI. El inicio de la demencia puede crear nuevos desafíos para los familiares debido a los cambios de comportamiento y las pérdidas en las habilidades de autonomía en los cuidados. Esto puede provocar que los miembros de la familia no estén seguros de qué apoyos pueden estar disponibles o cómo acceder a la información que se necesita, particularmente a medida que avanza la demencia (Jokinen et al., 2017). Se hicieron tres recomendaciones: (a) incluir un enfoque en brindar apoyo útil a familias adaptadas a los valores, creencias, etnicidad y circunstancias de la familia; (b) ayudar con la planificación para que las familias puedan decidir los mejores cursos de acción, y (c) permitir medios de reducción del estrés para mitigar los aspectos negativos del cuidado.

\section{Comentario}

La importancia de la Cumbre y sus esfuerzos se caracteriza por su oportunidad, la profundidad de los debates sobre cuestiones relevantes, su productividad y su estrategia de difusión de resultados, como informes y publicaciones revisadas por pares fácilmente accesibles a través de Internet. En conjunto abordan una diversidad de temas relevantes para los trabajadores en DI, envejecimiento, geriatría, demencia y atención social, y pueden influir en la planificación nacional, las políticas públicas, la práctica clínica, el desarrollo de servicios y la investigación. La Cumbre mostró que si bien hay elementos comunes entre la demencia y la atención de la demencia asociada a la DI y aquella de la población general, es necesaria una especialización adicional en el caso de la DI. Las principales implicaciones de política pública derivadas de esta cumbre incluyen una mayor atención a grupos de personas con alto riesgo de demencia; la inclusión de la población de impacto dentro de la planificación nacional para la demencia; revisiones cuidadosas de programas y servicios existentes para determinar qué barreras pueden estar presentes para la vida comunitaria continua cuando la demencia está presente; un mayor enfoque en la capacitación y educación del personal 
que trabaja con personas afectadas por demencia; mayor y mejor comunicación con personas que tienen DI y se ven afectados por la demencia; y la creación de opciones viables para la atención comunitaria de personas con demencia. La Cumbre fue un ejercicio productivo y debe agregar valor a la literatura sobre demencia e identificación, ayudar a aumentar la atención prestada a este tema y ayudar en el desarrollo e investigación de servicios.

\section{Referencias bibliográficas}

Alzheimer's Disease International (2015). World Alzheimer Report 2015: The global impact of dementia an analysis of prevalence, incidence, cost and trend. Recuperado de: https:// www.alz.co.uk/research/WorldAlzheimerReport2015.pdf.

Dodd, K., Watchman, K., Janicki, M. P., Coppus, A., Gaertner, C., Fortea, J., Santos, F. H., Keller, S. M., Strydom, A. y el Post-Diagnostic Supports Working Group (2017, en prensa). Consensus statement of the international summit on intellectual disability and dementia related to post-diagnostic support. Aging and Mental Health, Sep 7:1-10. doi: 10.1080/13607863.2017.1373065. [Epub ahead of print].

Janicki, M. P., McCallion. P., Splaine, M., Santos, F. H., Keller, S. M. y Watchman, K. (2017). Consensus statement of the international summit on intellectual disability and dementia related to nomenclature. Intellectual and Developmental Disabilities, 55 (5), 338346. doi: 10.1352/1934-9556-55.5.338.

Jokinen, N. S., Gomiero, T., Hogan, M., Larsen, F., Beránková, A., Santos, F. H., WatchMAN, K., JANICKI, M. P. y CrOw, J. (2017, in submission). Challenges and perspectives in the caregiving of ageing people with intellectual disability affected by dementia: Commentary from the International Summit on Intellectual Disability and Dementia.

McCallion, P., Hogan, M., Santos, F. H., McCarron, M., Service, K., Stemp, S., Keller, S., Fortea, J., Bishop, K., Watchman, K., Janicki, M. P. y Working Group of the International Summit on Intellectual Disability and Dementia (2017). Consensus statement of the international summit on intellectual disability and dementia related to end-of-life care in advanced dementia. Journal of Applied Research in Intellectual Disability, 30 (6), 1160-1164. doi: 10.1111/jar.12349.

McCarron, M., McCallion, P., Watchman, K., Janicki, M. P., Coppus, A., Service, K., Fortea, J., Hogan, M., Reilly, E. y Stemp, S. (2017a, in submission). Quality care for people with intellectual disability and advanced dementia: guidance on service provision.

McCarron, M., Stemp, S., Coppus, A., Fortea, J., Janicki, M. P., Reilly, E., Watchman, K., McCallion, P. y Summit Working Group on Advanced Dementia (2017b, in press). Defining advanced dementia in people with Down syndrome and other intellectual disabilities: consensus statement of the International Summit on Intellectual Disability and Dementia. Journal of Palliative Medicine.

Service, K. P., Hogan, M., Janicki, M. P., Cadovius, N., Watchman, K. y Beránková, A. (2017). Dying well with an intellectual disability and dementia. Journal of Dementia Care, 25 (4), 22-25.

Watchman, K., Janicki, M. P., Hogan, M., Udell, L., Quinn, S. y Beránková, A. (2017a, en prensa). Consensus statement of the international summit on intellectual disability and dementia on valuing the perspectives of persons with intellectual disability. Journal of Intellectual Disabilities. 
Watchman, K., Janicki, M. y miembros del International Summit on Intellectual DiSABILITY AND Dementia (2017b, en prensa). The intersection of intellectual disability and dementia: Report of the international summit on intellectual disability and dementia. The Gerontologist.

Watchman, K., Janicki, M. P., Splaine, M., Larsen, F. K., Gomiero, T. y Lucchino, R. (2017c). International summit consensus statement: Intellectual disability inclusion in national dementia plans. American Journal of Alzheimer's Disease and Other Dementias, 32 (4), 230-237, doi: 10.1177/1533317517704082.

World Health Organization (WHO) (2012). Dementia: A public health priority. Geneva, Switzerland: World Health Organization and Alzheimer's Disease International.

World Health Organization (WHO) (2017). Draft global action plan on the public health response to dementia. Recuperado de: http://www.who.int/mental_health/neurology/dementia/action_plan_consultation/en/. 